\title{
Calculation of Spectral Darkening and Visibility Functions for Solar Oscillations
}

\author{
C. Nutto $\cdot$ M. Roth $\cdot$ Y. Zhugzhda $\cdot$ J. Bruls $\cdot$ \\ O. von der Lühe
}

Received: 4 October 2007 / Accepted: 25 January 2008 / Published online: 15 February 2008

(C) Springer Science+Business Media B.V. 2008

\begin{abstract}
Calculations of spectral darkening and visibility functions for the brightness oscillations of the Sun resulting from global solar oscillations are presented. This has been done for a broad range of the visible and infrared continuum spectrum. The procedure for the calculations of these functions includes the numerical computation of depth-dependent derivatives of the opacity caused by $p$ modes in the photosphere. A radiative-transport code was used for this purpose to get the disturbances of the opacities from temperature and density fluctuations. The visibility and darkening functions are obtained for adiabatic oscillations under the assumption that the temperature disturbances are proportional to the undisturbed temperature of the photosphere. The latter assumption is the only way to explore any opacity effects since the eigenfunctions of $p$-mode oscillations have not been obtained so far. This investigation reveals that opacity effects have to be taken into account because they dominate the violet and infrared part of the spectrum. Because of this dominance, the visibility functions are negative for those parts of the spectrum. Furthermore, the darkening functions show a wavelength-dependent change of sign for some wavelengths owing to these opacity effects. However, the visibility and darkening functions under the assumptions used contradict the observations of global $p$-mode oscillations, but it is beyond doubt that the opacity effects influence the brightness fluctuations of the Sun resulting from global oscillations.
\end{abstract}

Helioseismology, Asteroseismology, and MHD Connections Guest Editors: Laurent Gizon and Paul Cally.

C. Nutto $(\varangle) \cdot$ Y. Zhugzhda · J. Bruls · O. von der Lühe

Kiepenheuer-Institut für Sonnenphysik, Schöneckstraße 6, 79104, Freiburg, Germany

e-mail: nutto@kis.uni-freiburg.de

M. Roth

Max-Planck-Institut für Sonnensystemforschung, 37191, Katlenburg-Lindau, Germany

Y. Zhugzhda

Institute of Terrestrial Magnetism, Ionosphere and Radio Wave Propagation of the Russian Academy of

Sciences, Troitsk, Moscow Region 142092, Russia 
Keywords Oscillations: Solar · Waves: Acoustic · Integrated Sun observations · Helioseismology: Theory $\cdot$ Spectrum: Continuum

\section{Introduction}

Since the ACRIM experiment (Woodard, 1984) it has been well known that solar oscillations lead to brightness fluctuations of the Sun as a star. However, the spatial integration of the flux disturbances over the whole solar disk suppresses high-frequency modes, and photometric observations of the Sun as a star can only resolve spatial oscillation modes with low harmonic degree $(\ell<3)$. But if the visibility of these low-degree modes is suppressed at some positions on the solar disk through opacity effects, then it might be possible to observe oscillation modes with harmonic degree $\ell \geq 3$.

The space experiment IPHIR onboard the Phobos spacecraft (Fröhlich et al., 1988) showed that the amplitudes of the brightness fluctuations resulting from $p$ modes are different for different optical wavelengths. Zhugzhda, Dzhalilov, and Staude (1993) showed with the consideration of nonadiabatic waves in a uniform, nongray atmosphere that this problem cannot be solved with the introduction of the Rosseland mean opacity. With the calculation of visibility functions for low-degree nonradial oscillations and a comparison with IPHIR data, Toutain and Gouttebroze (1993) suggested two limiting cases for the explanation of the origin of the brightness fluctuations:

- Intensity and flux perturbations, caused by adiabatic oscillations, are driven by opacity effects and thus have to be taken into account for calculations of visibility functions.

- The visibility functions depend on nonadiabatic effects, and opacity disturbances can be neglected (blackbody approximation).

The direct way to solve the problem is to solve the eigenproblem for nonadiabatic oscillations for a solar model that includes a standard model of the photosphere. To our knowledge nobody has done this so far since the set of integro-differential equations of radiative hydrodynamics has to be solved. Staude, Dzhalilov, and Zhugzhda (1994) and Zhugzhda, Staude, and Bartling (1996) proposed using another approach in which the temperature and density fluctuations resulting from the $p$ modes are assumed to be given. Under some special assumptions, this makes it possible to obtain so-called darkening functions, which show the dependence of brightness fluctuations with respect to the position on the solar disk. The intention was to develop a qualitative explanation of brightness oscillations to improve observations of solar oscillations. The different wavelengths under consideration indicated that the brightness fluctuations of the most dominant oscillation mode $(\ell=0)$ vanishes for certain positions on the solar disk (e.g., the darkening function changes its sign). With the assumption that these calculations can be easily extended to modes with harmonic degree $0<\ell<3$ and that those functions will also show a change of sign, modes with higher harmonic degree $(\ell \geq 3)$ might be observable.

Zhugzhda, Staude, and Bartling (1996) carried out calculations of darkening functions for a few wavelengths only. However, the visibility functions were not calculated. In this paper the calculations of darkening and visibility functions are performed for a wide range of wavelengths, from the near UV to the infrared, to see whether the opacity effects are essential for this range of the spectrum. This investigation will help us to analyze the observations from photometric instruments such as DIFOS onboard CORONAS-F (Lebedev et al., 2004). 


\section{Intensity and Flux Fluctuations from $p$ Modes}

To avoid the full solution of the equations of radiation hydrodynamics (Zhugzhda, Staude, and Bartling, 1996) we expand the general expression for the intensity in a series around the equilibrium state up to the first order of small disturbances of the opacity. Under the assumption of local thermodynamic equilibrium (LTE), the fluctuations of the emergent intensity $\left(\delta I_{v}\right)$ normalized to the specific intensity $\left(I_{0 v}\right)$ at the center of the solar disk are (Zhugzhda, Staude, and Bartling, 1996)

$$
\begin{aligned}
\frac{\delta I_{v}}{I_{0 v}}= & \int_{0}^{\infty} \mathrm{e}^{-\tau_{v} / \mu}\left[\frac{\mathrm{d} B_{v}\left(\tau_{v}\right)}{\mathrm{d} \ln T_{0}} \frac{\delta T}{T_{0}}+\frac{\delta \kappa_{v}}{\kappa_{0 v}}\left(B_{v}\left(\tau_{v}\right)-I_{v}\left(\tau_{v}, \mu\right)\right)\right] \frac{\mathrm{d} \tau_{v}}{\mu} \\
& \times\left[\int_{0}^{\infty} \mathrm{e}^{-\tau_{v}} B_{v}\left(\tau_{v}\right) \mathrm{d} \tau_{v}\right]^{-1},
\end{aligned}
$$

where $\tau_{v}$ is the optical depth at the frequency $\nu, B_{v}$ is the Planck function, $\mu=\cos \theta$, and $\theta$ is the polar angle. The unperturbed, outgoing intensity, originating at optical depth $\tau_{\nu}$, is

$$
I_{\nu}\left(\tau_{v}, \mu\right)=\int_{\tau_{v}}^{\infty} \mathrm{e}^{-\left(\tau_{\nu}^{\prime}-\tau_{\nu}\right) / \mu} B_{\nu}\left(\tau_{v}^{\prime}\right) \frac{\mathrm{d} \tau_{v}^{\prime}}{\mu} .
$$

The fluctuation of the opacity $\left[\delta \kappa_{v}(\rho, T)\right]$ is given by the fluctuation of the density $(\delta \rho)$ and the temperature $(\delta T)$,

$$
\frac{\delta \kappa_{v}}{\kappa_{0 v}}=\frac{\mathrm{d} \ln \kappa_{0 v}}{\mathrm{~d} \ln T} \frac{\delta T}{T_{0}}+\frac{\mathrm{d} \ln \kappa_{0 v}}{\mathrm{~d} \ln \rho} \frac{\delta \rho}{\rho_{0}} .
$$

Equation (1) considers the various contributions to the intensity fluctuations. The first term appears because of the temperature fluctuation in the source function, which can be described by the Planck function in the case of LTE. The second term appears because of the perturbation of the opacity from disturbances in density and temperature. An approximation for the temperature and the density perturbations will be used. The solution yields the intensity fluctuation of a nongray atmosphere caused by small disturbances. This can be used for the disturbances caused by $p$ modes since they are small enough and the linear approximation works perfectly.

To simplify Equation (1) it is assumed that disturbances of the equilibrium state are adiabatic. In this special case the temperature and density disturbances are connected by the simple relation

$$
\frac{\delta T}{T}=\left(\Gamma_{3}-1\right) \frac{\delta \rho}{\rho},
$$

where $\Gamma_{3}$ is the third adiabatic exponent. For a neutral or fully ionized gas it is constant and has the value $\Gamma_{3}-1=2 / 3$, whereas for a partially ionized gas it can reach values of $\Gamma_{3}-1 \leq 0.1$. The first case is given for the photosphere, which is completely dominated by neutral hydrogen. The second case applies for the deeper photosphere and the chromosphere, where the gas is partially ionized.

In the case of adiabatic disturbances, the intensity fluctuations depend only on temperature fluctuations and on parameters of the quiet undisturbed photosphere. The dependence of the intensity fluctuations on $\mu$ is a darkening function for the adiabatic disturbances. To obtain the darkening function for $p$ modes, the dependence of the temperature disturbances on depth $\left[\delta T\left(\tau_{v}\right)\right]$ has to be substituted into Equation (1). But this function is not known, 
even for adiabatic disturbances. To make a qualitative analysis of the opacity effects we simplify the problem by assuming that the disturbances are proportional to their undisturbed values (Zhugzhda, Staude, and Bartling, 1996),

$$
\frac{\delta T}{T_{0}}=\text { constant }
$$

which is a rather crude approximation. It holds only for continuum radiation that originates from a thin layer of the solar atmosphere. With this assumption the relative disturbances $\delta T / T_{0}$ and $\delta \rho / \rho_{0}$ are independent of the optical depth $\left(\tau_{v}\right)$ and can be factored out of the integral. Now, with relationship (4) the relative intensity variation can be described in units of $\delta \rho / \rho$ or $\delta T / T$. This leads to the darkening function

$$
\Delta_{v}=\frac{\delta I_{v} / I_{0 v}}{\delta T / T_{0}}
$$

which in our case is expressed in units of $\delta T / T_{0}$.

To obtain the visibility function, the darkening function has to be integrated over the entire solar disk. This yields the following expression for the flux perturbation for the most dominant mode $\ell=0$ :

$$
\begin{aligned}
\frac{\delta F_{v} / F_{0 v}}{\delta T / T_{0}}= & \left\{\int _ { 0 } ^ { \infty } \left[\frac{\partial B_{v}\left(\tau_{v}\right)}{\partial \ln T} E_{2}\left(\tau_{v}\right)\right.\right. \\
& -\left(\frac{\partial \ln \kappa_{v}}{\partial \ln T}\right)_{\rho} \int_{\tau_{v}}^{\infty} \frac{\mathrm{d} B_{v}\left(\tau_{v}^{\prime}\right)}{\mathrm{d} \tau_{v}^{\prime}} E_{2}\left(\tau_{v}^{\prime}\right) \mathrm{d} \tau_{v}^{\prime} \\
& \left.\left.-\frac{1}{\Gamma_{3}\left(\tau_{v}\right)-1}\left(\frac{\partial \ln \kappa_{v}}{\partial \ln \rho}\right)_{T} \int_{\tau_{v}}^{\infty} \frac{\mathrm{d} B_{v}\left(\tau_{v}^{\prime}\right)}{\mathrm{d} \tau_{v}^{\prime}} E_{2}\left(\tau_{v}^{\prime}\right) \mathrm{d} \tau_{v}^{\prime}\right] \mathrm{d} \tau_{v}\right\} \\
& /\left\{\int_{0}^{\infty} B_{v}\left(\tau_{v}\right) E_{2}\left(\tau_{v}\right) \mathrm{d} \tau_{v}\right\} .
\end{aligned}
$$

\section{Numerical Calculation of Darkening and Visibility Functions for Solar Oscillations with Degree $\ell=0$}

For the evaluation of the darkening functions one needs to know radiative transport quantities for the solar atmosphere as functions of the optical depth $\tau_{v}$. For this purpose the radiative transport code $\mathrm{RH}^{1}$ was used (Uitenbroek, 2001). The code is based on the MALI (Multi-level Approximate Lambda Iteration) formalism by Rybicki and Hummer (1991). It solves the combined equations of statistical equilibrium and radiative transport under the general assumption of non-LTE conditions for a multilevel atom in a given plasma. In our case we choose hydrogen to be the considered atom. In addition to opacities and emissivities from the transitions in the hydrogen atom, the code accounts for background radiation sinks and sources from other atoms and molecules and all relevant continuum processes such as $\mathrm{H}^{-}$bound-bound and bound-free processes, Rayleigh scattering off neutral hydrogen, Thompson scattering off free electrons, helium, and $\mathrm{H}_{2}$, and hydrogen free-free processes. The atoms and molecules considered for the background radiation and opacities are listed

\footnotetext{
${ }^{1}$ http://www.nso.edu/staff/uitenbr/rh.html.
} 
Table 1 Atoms and molecules that are considered as background elements by the radiative transport code.

\begin{tabular}{ll}
\hline Atoms & $\mathrm{He}, \mathrm{C}, \mathrm{N}, \mathrm{O}, \mathrm{Na}, \mathrm{Mg}, \mathrm{Al}, \mathrm{Si}, \mathrm{S}, \mathrm{Ca}, \mathrm{Fe}$ \\
Molecules & $\mathrm{H}_{2}, \mathrm{H}_{2}^{+}, \mathrm{C}_{2}, \mathrm{~N}_{2}, \mathrm{O}_{2}, \mathrm{CH}, \mathrm{CO}, \mathrm{CN}, \mathrm{NH}, \mathrm{NO}, \mathrm{OH}, \mathrm{H}_{2} \mathrm{O}$ \\
\hline
\end{tabular}

in Table 1. In addition, it is necessary to provide the radiative transport code with a model of the solar atmosphere. We choose the semi-empirical, one-dimensional, quiet-Sun model FAL-C by Fontenla et al. (1999).

For the calculation of the darkening function we are interested not only in the radiative quantities alone but also in their disturbances. Considering Equation (1), we need the disturbances of the Planck function from temperature variations and the disturbances of the opacity from variation of temperature and density.

To calculate the derivatives

$$
\left(\frac{\partial B_{v}}{\partial \ln T}\right), \quad\left(\frac{\partial \ln \kappa_{v}}{\partial \ln T}\right)_{\rho}, \quad \text { and } \quad\left(\frac{\partial \ln \kappa_{v}}{\partial \ln \rho}\right)_{T},
$$

we disturb the quantities $T$ and $\rho$ of the atmospheric model; these are then used as input for the radiative transport code. We use temperature disturbances of $-5 \mathrm{~K}$ and $+5 \mathrm{~K}$, which is less then $1 \%$ of the undisturbed temperature of the atmosphere, and the derivatives behave linearly under these small temperature disturbances. With Equation (4) for the adiabatic relation, it can be assumed that the relative disturbances of the density are around $1 \%$. Thus, the hydrogen and electron populations are modified accordingly in the model of the atmosphere. While one modification is applied, all other values are kept constant. This procedure allows us to calculate the partial derivatives of the Planck function and the opacity using the centered-difference approximation (Pozrikidis, 1998).

Our interest is in the calculation of the darkening and visibility functions for a broad range of the visible and infrared spectrum. This has been done for 79 wavelengths between 250 and $2100 \mathrm{~nm}$. We have to avoid any lines since we assume a temperature disturbance that is constant (see Equation (5)). This is only justified as long as the radiation originates from a thin layer. This applies for the continuum radiation, but spectral lines form in higher layers and are not constrained to a small part of the solar atmosphere.

\section{Results}

\subsection{Derivatives}

First, the derivatives of the Planck function $\left(\partial B_{v} / \partial \ln T\right)$ were calculated. The Planck function has the advantage that the derivative can be evaluated analytically and the numerical values can be compared with the analytical function. A comparison showed that the numerical calculation of the derivatives coincides with the analytical values within a few tenths of a percent.

In Figure 1 the derivative of the opacity with respect to temperature is shown as a function of wavelength $\lambda$ and monochromatic optical depth $\tau_{v}$. Although the model atmosphere extends from the photosphere to the transition region, we restrict ourselves to the photosphere since we are only interested in continuum radiation. Certain discontinuities in the plot are noticeable. The corresponding wavelengths coincide with the ionization wavelengths of neutral hydrogen, namely the Balmer continuum $(\lambda=364.6 \mathrm{~nm})$. Just above the Balmer continuum there is another visible ionization edge that belongs to magnesium with $\lambda=375.6 \mathrm{~nm}$. 


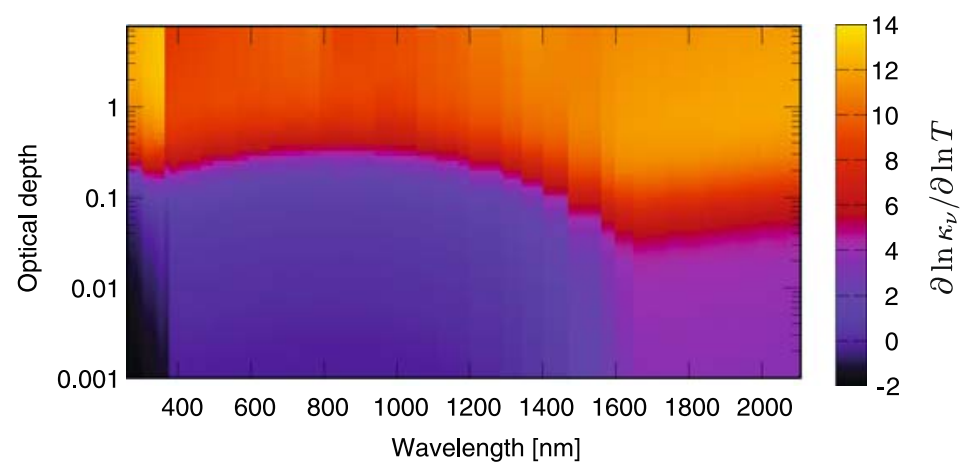

Figure 1 Derivative of opacity with respect to temperature as a function of wavelength and the monochromatic optical depth $\tau_{v}$.

These discontinuities can be explained with the ionization setting in abruptly at wavelengths just smaller than the ionization edges. If we assume positive temperature disturbances, then higher temperatures give rise to a higher ionization rate, which causes a greater increase of the opacity. Thus, the derivative of the opacity is greater for wavelengths shorter than the ionization edges. The effect diminishes with smaller optical depth, since the influence of neutral hydrogen on the opacity vanishes for higher parts of the atmosphere (Vernazza, Avrett, and Loeser, 1976). The influence of neutral hydrogen causes the steep rise of the derivative for the deeper layers. For higher layers, the derivative is dominated by the influence of $\mathrm{H}^{-}$ions (Vernazza, Avrett, and Loeser, 1976) and the opacity is less sensitive to temperature disturbances. This can be explained by the combined effect of the destruction of $\mathrm{H}^{-}$ions from higher temperatures and the higher ionization of neutral hydrogen, which results in a higher electron density. Investigations showed that if those higher electron densities are not taken into account the derivatives turn out to be negative for those parts of the spectrum where the opacity is dominated by $\mathrm{H}^{-}$. In conclusion, the creation of $\mathrm{H}^{-}$resulting from a higher electron density wins over the destruction of $\mathrm{H}^{-}$resulting from higher temperatures and thus the opacity increases. There is also a change of the behavior of the derivative for $\lambda=1645 \mathrm{~nm}$. This can be explained by the fact that above this wavelength the free-free processes of $\mathrm{H}^{-}$start to dominate the contribution to the opacity.

The results for the derivative of the opacity with respect to the density are shown in Figure 2. First, we can point out the discontinuities at the continuum edges again, where the same explanation as for Figure 1 applies. For deeper layers, the discontinuities for the Paschen continuum $(\lambda=820.4 \mathrm{~nm})$ and the Brackett continuum $(\lambda=1458.4 \mathrm{~nm})$ of neutral hydrogen are more pronounced than for the derivative of the opacity with respect to temperature.

Knowing the derivatives of opacity, we can now calculate the darkening and visibility functions for the most dominant solar oscillation mode with $\ell=0$.

\subsection{Darkening and Visibility Functions}

In Figure 3 the darkening functions $\left(\Delta_{v}\right)$ (see Section 2) are shown for selected wavelengths. Some of the these wavelengths coincide with the wavelengths examined by Zhugzhda, Staude, and Bartling (1996) and a comparison ${ }^{2}$ of the results for those wavelengths shows

${ }^{2}$ In Zhugzhda, Staude, and Bartling (1996) the graphs for $\lambda=275 \mathrm{~nm}$ and $\lambda=1645 \mathrm{~nm}$ should be switched. 


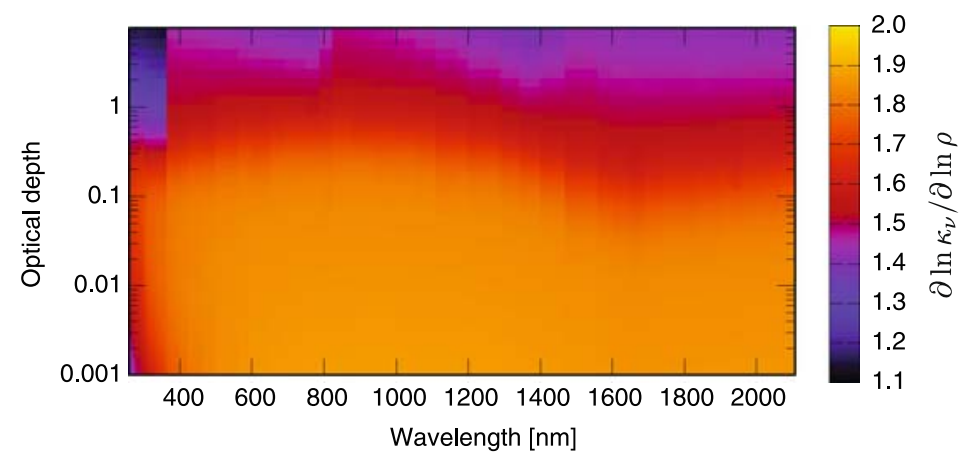

Figure 2 Derivative of opacity with respect to density as a function of wavelength and optical depth.

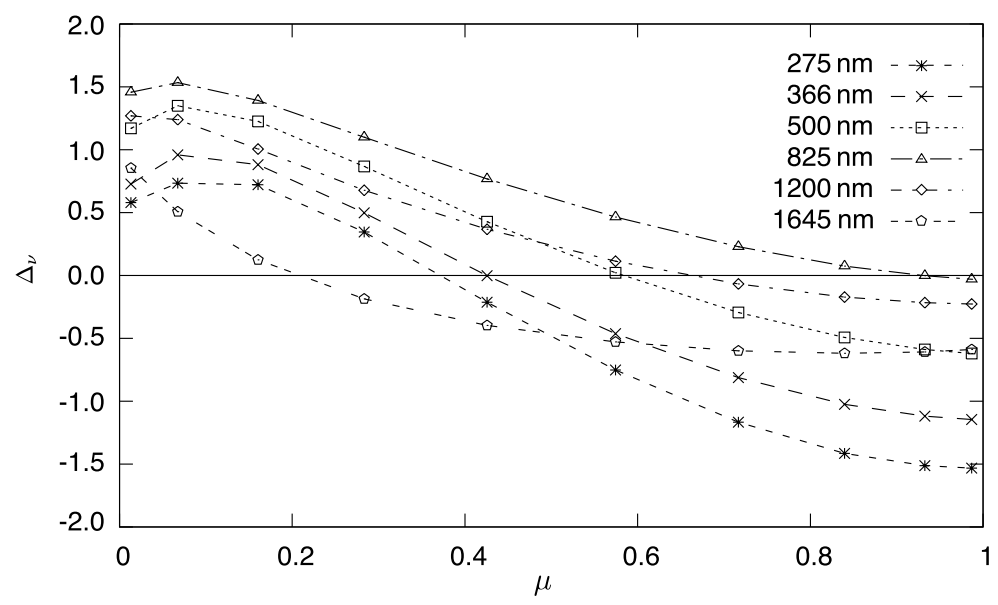

Figure 3 The relative darkening functions $\left(\Delta_{\nu}\right)$ for selected wavelengths versus position on the solar disk $(\mu=\cos \theta)$. The marks show the sampling points on the solar disk.

that they are similar, although there are some small differences in the position of the change of sign. This might be caused by a small difference in the derivatives of the opacity derived in this paper and the derivatives used by Zhugzhda, Staude, and Bartling (1996) for higher layers of the atmosphere. The wavelengths displayed show that the position of the change of sign on the solar disk depends strongly on the wavelength. For the wavelengths whose opacity is mostly dominated by the $\mathrm{H}^{-}$ions $(e . g ., 825 \mathrm{~nm})$, the position of the change of sign of the darkening function is almost at the center of the solar disk whereas the function increases toward the limb of the Sun. Thus, for this particular wavelength the oscillation mode $\ell=0$ would be best observed at the limb while it almost vanishes at the center of the disk. To get the visibility of global oscillation modes for each wavelength, the darkening functions have to be integrated over the solar disk. This can be done by either numerical integration of the darkening function for each wavelength or by direct evaluation of Equation (7). We choose the latter option for the calculation of the monochromatic visibility function.

The result of this integration can be seen in Figure 4, where the absolute visibility function $\left[\delta F_{v} /\left(\delta T / T_{0}\right)\right]$ is plotted together with its different contributions from the Planck function and opacity. This plot clearly indicates that the visibility functions are dominated by 


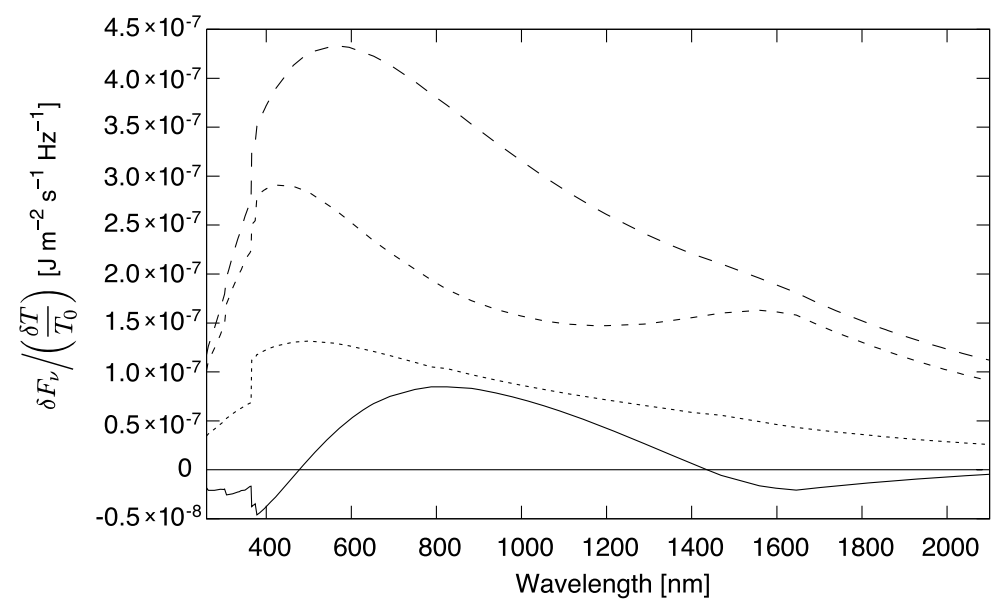

Figure 4 The absolute visibility function versus wavelength (solid line). In addition, the different contributions $\partial B_{\nu} / \partial \ln T$ (long-dashed line), $\partial \ln \kappa_{\nu} / \partial \ln T$ (medium-dashed line), and $\partial \ln \kappa_{\nu} / \partial \ln \rho$ (short-dashed line) originating from the Planck function and the opacity are shown.

the effects of the opacity. This is due to the assumption of adiabatic oscillations for which the temperature and density disturbances are in phase. The effect of the oscillation on the opacity is big enough to overcome the disturbances of the Planck function and for shorter and longer wavelengths positive disturbances actually result in a darkening of the solar flux. Considering the visibility function, one sees that the oscillation mode $\ell=0$ has the biggest influence on the disturbance of the flux at wavelengths around $\lambda=800 \mathrm{~nm}$. Since the visibility functions also show a change of sign for two wavelengths of the considered spectrum, it is interesting to note that for $\lambda=480 \mathrm{~nm}$ and $\lambda=1450 \mathrm{~nm}$ the global oscillation of mode $\ell=0$ should not be visible. This can actually be used as a test for the assumption of adiabatic oscillations, as will be discussed in the following.

\section{Conclusions}

There is an essential distinction between visibility functions for the global solar and stellar oscillations observed by measurements of Doppler shifts and continuum brightness fluctuations. In the case of Doppler shifts the visibility is defined by the projection of the velocity vector of the oscillation modes onto the line of sight. Thus, it is not complicated to calculate the visibility function for every spherical harmonic.

The derivation of the visibility functions for brightness fluctuations resulting from global oscillations is more difficult. We have succeeded in obtaining the spectral darkening and visibility functions over a broad range of the visible and infrared spectrum of the Sun. Two simplifications of the problem make this possible: the adiabatic approximation and the condition $\delta T / T_{0}=$ constant.

We found that the brightness fluctuations are not necessarily proportional to temperature fluctuations. The darkening function shows a change of sign and the visibility function can be negative (e.g., the brightness can decrease with the increase of the temperature) owing to radiative flux blocking.

However, there are some problems considering the visibility function. In accordance with Figure 4, the brightness fluctuations should be absent for 480 and $1450 \mathrm{~nm}$. But this is not 
the case. Brightness fluctuations are observed at those wavelengths by SOHO/VIRGO and CORONAS/DIFOS. This clearly indicates that one of the assumptions is not correct, if not both of them. The condition with constant temperature disturbances should be relaxed in general. It is necessary to know how the temperature fluctuations depend on the depth in the solar atmosphere.

The second assumption in use is the adiabatic approximation. Our calculations confirm one of the results of Toutain and Gouttebroze (1993). If adiabatic conditions are assumed, the opacity perturbations show a great influence on the visibility functions. But, as already mentioned, in this case the brightness fluctuations should not be visible for 480 and $1450 \mathrm{~nm}$, which is not in accordance with observations. Thus, this approximation is violated in the solar photosphere. Zhugzhda (2006) discovered that there are phase shifts between brightness fluctuations in different spectral channels of the DIFOS photometric instrument. This is only possible if oscillations are nonadiabatic since in this case there is a phase shift between the temperature and density fluctuations. Moreover, the nonadiabatic $p$ modes are coupled with temperature waves (Zhugzhda, 1983). Consequently, the darkening and visibility functions for nonadiabatic oscillations are complex functions. Multichannel observations of CORO$N A S$ together with SOHO observations make it possible to explore these nonadiabatic oscillations.

The visibility and darkening functions are needed for the interpretation of the observations of solar and stellar observations. The main result of the current exploration is that the effect of the opacity fluctuations on brightness oscillations dominates. However, it is a rather complicated problem to find the correct functions for adiabatic and nonadiabatic oscillations. The current paper just presents the first step on this road.

Acknowledgements YZ is grateful to RFFI (Grant No. 06-02-16359). CN, MR, YZ and OvdL acknowledge support from the European Helio- and Asteroseismology Network-HELAS. HELAS is funded by the European Union's Sixth Framework programme. We also thank J. Staude for his helpful comments on our research. We thank H. Uitenbroek for the provision of his radiative transport code RH. Furthermore, we gratefully thank the anonymous referee for the substantial revision and valuable advice that helped to improve this paper.

\section{References}

Fontenla, J., White, O.R., Fox, P.A., Avrett, E.H., Kurucz, R.L.: 1999, Calculation of Solar irradiances. I. Synthesis of the Solar spectrum. Astrophys. J. 518, 480-499.

Fröhlich, C., Bonnet, R.M., Bruns, A.V., Delaboudinière, J.P., Domingo, V., Kotov, V.A., Kollath, Z., Rashkovsky, D.N., Toutain, T., Vial, J.C.: 1988, IPHIR: The helioseismology experiment on the PHOBOS mission. In: Rolfe, E.J. (ed.) Seismology of the Sun and Sun-Like Stars, ESA-SP 286, European Space Agency, Noordwijk, 359-362.

Lebedev, N.I., Kuznetsov, V.D., Oraevskii, V.N., Staude, J., Kostyk, R.I.: 2004, The helioseismological CORONAS-F DIFOS experiment. Astron. Rep. 48, 871 -875. doi:10.1134/1.1809399.

Pozrikidis, C.: 1998, Numerical Computation in Science and Engineering, Oxford University Press, New York.

Rybicki, G.B., Hummer, D.G.: 1991, An accelerated lambda iteration method for multilevel radiative transfer. I. Non-overlapping lines with background continuum. Astron. Astrophys. 245, $171-181$.

Staude, J., Dzhalilov, N.S., Zhugzhda, Y.D.: 1994, Radiation-hydrodynamic waves and global solar oscillations. Solar Phys. 152, $227-239$.

Toutain, T., Gouttebroze, P.: 1993, Visibility of solar p-modes. Astron. Astrophys. 268, 309-318.

Uitenbroek, H.: 2001, Multilevel radiative transfer with partial frequency redistribution. Astrophys. J. 557, 389 - 398. doi:10.1086/321659.

Vernazza, J.E., Avrett, E.H., Loeser, R.: 1976, Structure of the solar chromosphere. II. The underlying photosphere and temperature-minimum region. Astrophys. J. Suppl. 30, 1-60. 
Woodard, M.: 1984, Observations of low-degree modes from the solar maximum mission (extended abstract). In: Ulrich, R.K., Harvey, J., Rhodes, E.J. Jr., Toomre, J. (eds.) Solar Seismology from Space, JPL, Pasadena, $195-197$.

Zhugzhda, Y.D.: 1983, Non-adiabatic oscillations in an isothermal atmosphere. Astrophys. Space Sci. 95, 255-275.

Zhugzhda, Y.D.: 2006, Analytical signal as a tool for studying solar p-modes. Astron. Lett. 32, 329 - 343.

Zhugzhda, Y.D., Dzhalilov, N.S., Staude, J.: 1993, Radiation-hydrodynamic waves in an optically non-grey atmosphere. Astron. Astrophys. 278, L9-L12.

Zhugzhda, Y.D., Staude, J., Bartling, G.: 1996, Spectral darkening functions of solar $p$-modes - an effective tool for helioseismology. Astron. Astrophys. 305, L33 - L36. 